Louisiana State University

LSU Digital Commons

$1-1-1999$

\title{
Quantum-path analysis and phase matching of high-order harmonic generation and high-order frequency mixing processes in strong laser fields
}

\author{
Philippe Balcou \\ École polytechnique \\ Anne S. Dederichs \\ École polytechnique \\ Mette B. Gaarde \\ École polytechnique \\ Anne L'Huillier \\ École polytechnique
}

Follow this and additional works at: https://digitalcommons.Isu.edu/physics_astronomy_pubs

\section{Recommended Citation}

Balcou, P., Dederichs, A., Gaarde, M., \& L'Huillier, A. (1999). Quantum-path analysis and phase matching of high-order harmonic generation and high-order frequency mixing processes in strong laser fields. Journal of Physics B: Atomic, Molecular and Optical Physics, 32 (12), 2973-2989. https://doi.org/10.1088/ 0953-4075/32/12/315 


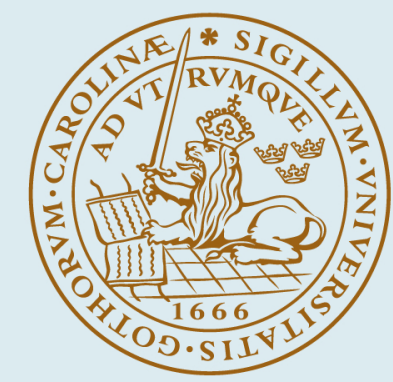

\title{
LUND UNIVERSITY
}

\section{Quantum-path analysis and phase matching of high-order harmonic generation and high-order frequency mixing processes in strong laser fields}

\author{
Balcou, P; Dederichs, A. S; Gaarde, Mette; L'Huillier, Anne \\ Published in: \\ Journal of Physics B: Atomic, Molecular and Optical Physics
}

DOI:

10.1088/0953-4075/32/12/315

1999

Link to publication

Citation for published version (APA):

Balcou, P., Dederichs, A. S., Gaarde, M., \& L'Huillier, A. (1999). Quantum-path analysis and phase matching of high-order harmonic generation and high-order frequency mixing processes in strong laser fields. Journal of Physics B: Atomic, Molecular and Optical Physics, 32(12), 2973-2989. https://doi.org/10.1088/0953$4075 / 32 / 12 / 315$

Total number of authors:

4

\footnotetext{
General rights

Unless other specific re-use rights are stated the following general rights apply:

Copyright and moral rights for the publications made accessible in the public portal are retained by the authors and/or other copyright owners and it is a condition of accessing publications that users recognise and abide by the legal requirements associated with these rights.

- Users may download and print one copy of any publication from the public portal for the purpose of private study or research.

- You may not further distribute the material or use it for any profit-making activity or commercial gain

- You may freely distribute the URL identifying the publication in the public portal

Read more about Creative commons licenses: https://creativecommons.org/licenses/

Take down policy

If you believe that this document breaches copyright please contact us providing details, and we will remove access to the work immediately and investigate your claim.
} 
Quantum-path analysis and phase matching of high-order harmonic generation and highorder frequency mixing processes in strong laser fields

This article has been downloaded from IOPscience. Please scroll down to see the full text article.

1999 J. Phys. B: At. Mol. Opt. Phys. 322973

(http://iopscience.iop.org/0953-4075/32/12/315)

View the table of contents for this issue, or go to the journal homepage for more

\section{Download details:}

IP Address: 130.235.188.104

The article was downloaded on 06/07/2011 at 09:48

Please note that terms and conditions apply. 


\title{
Quantum-path analysis and phase matching of high-order harmonic generation and high-order frequency mixing processes in strong laser fields
}

\author{
Philippe Balcou $\dagger$, Anne S Dederichs $\ddagger$, Mette B Gaarde $\ddagger \S$ and \\ Anne L'Huillier $\ddagger$ \\ † Laboratoire d'Optique Appliquée, ENSTA-Ecole Polytechnique, Unité Mixte de Recherches \\ 7639, Centre National de la Recherche Scientifique, F-91761 Palaiseau Cedex, France \\ \$ Department of Physics, Lund Institute of Technology, PO Box 118, S-221 00 Lund, Sweden \\ $\S$ Niels Bohr Institute, Ørsted Laboratory, Universitetsparken 5, 2100 Kbh Ø, Denmark
}

Received 17 November 1998

\begin{abstract}
We study phase-matching conditions for high-order harmonic generation as well as high-order sum- and difference-frequency mixing processes in strong laser fields, using a graphical approach described in Balcou et al (1997 Phys. Rev. A 55 3204-10). This method is based on the analysis of the different quantum paths that contribute, with different phase properties, to the single-atom response. We propose a simple numerical method to disentangle the quantum paths contributing to the generation process. We present graphical maps of the phase matching around the laser focus, which allow one to predict the geometries that optimize the conversion efficiency of the process considered. The method is applied to the study of sum- and difference-frequency mixing processes. The qualitative predictions of the graphical phase-matching approach are confirmed by numerical propagation calculations.
\end{abstract}

\section{Introduction}

The understanding of high-order harmonic generation (HHG) processes has progressed considerably over the past five years [1], largely owing to the development of the semiclassical interpretation [2,3]. In this approach, an electron initially in the ground state of an atom, and exposed to an intense, low-frequency, linearly polarized, electromagnetic field, first tunnels through the barrier formed by the Coulomb and the laser fields. When the laser field changes sign, the electron may be driven back towards the core with high kinetic energy and recombine to the ground state, giving rise to emission of high-energy harmonic photons. A quantummechanical formulation of this model, known as the strong-field approximation (SFA) [4,5], has been used extensively to describe different aspects of the generation process. In particular, it is used as input in a propagation calculation, and has proved very successful in modelling the properties of the emitted radiation.

In the semiclassical description, the sum of the ionization potential and incoming kinetic energy should match the energy of the emitted photon. For a given photon energy, there may be one, two or more classical trajectories fulfilling this requirement, corresponding, in a quantum framework, to different quantum paths. The dipole moment at a given harmonic frequency can be separated into a sum of contributions from these quantum paths: $d=\sum_{i} d_{i}$. The amplitude of each component $d_{i}$ varies approximately exponentially with the intensity of the generating 
laser field, whereas its phase is proportional to the laser intensity [6]. This phase behaviour can be understood heuristically as follows. In a quasi-classical model, the phase corresponds to the action $S$ of the electronic motion in the continuum. $S$ is approximately equal to the product of the electron average energy and the return time $\tau$ (defined as the time the electron spends in the continuum) [4]. The electron average energy, also called the ponderomotive energy, is simply proportional to the intensity, so that the action and the phase are also proportional to the intensity, with a coefficient depending on the release and recombination times of the electron in the continuum. The different quantum paths therefore contribute with different phase behaviours to the dipole moment. For a harmonic belonging to the plateau region, there are mainly two dominant quantum paths. The first one, with a short return time $\tau_{1}$, contributes with a phase that varies very slowly with the laser intensity whereas the second one, with a longer return time $\tau_{2}$, contributes with a rapidly decreasing phase [4].

When calculating the macroscopic response of an ensemble of atoms, phase matching between the generated fields and the laser-induced polarization (proportional to the dipole moment) is essential. Since the different quantum paths have different phase variations as a function of the laser intensity, and consequently different phase variations in the focal volume, they are not phase matched in the same geometrical conditions [7,8]. Depending on these conditions, one quantum path can be better phase matched than the other and vice versa. This has important consequences for the spatial and temporal coherence properties of the generated harmonic radiation.

In a recent paper, a graphical method to analyse phase matching of high-order harmonic generation [9] was proposed. This method is based on the interpretation of the spatial gradient of the atomic dipole phase as a wavevector, leading to a vectorial formulation of phase-matching conditions. The distributions for the atomic phase wavevectors and the field's wavevectors can be represented graphically, allowing one to identify the regions of good phase matching. It is also possible to consider separately phase matching for the different quantum paths contributing to harmonic generation. This has been done so far only with the high-harmonic dipole moments from the SFA model [4], because they could be calculated analytically, and because the quantum paths could be separated by solving saddle-point equations. It could be done more generally for any dipole moments (and, in particular, for those obtained numerically), provided one had a method to disentangle the quantum paths.

The aim of the present paper is therefore twofold. Firstly, we present a new numerical method to disentangle the contributing quantum paths from a dipole moment obtained numerically, for several laser peak intensities. This method is based on a wavelet-like slidingwindow Fourier transform. We apply this method to 45th harmonic generation of $800 \mathrm{~nm}$ radiation, calculated numerically using the SFA, and compare the results with those of the direct quasi-classical method. We also consider the influence of a second (weaker) laser field on the different quantum paths. This problem is related to a recent experiment [10] and theoretical analysis [11] of sum- and different-frequency mixing processes, occurring when an intense infrared laser field is mixed with the less intense, but tunable visible light from an optical parametric generator (OPG).

Secondly, we apply the graphical phase-matching method to the contributions from the different quantum paths. We investigate, in particular, the problem of sum- and differencefrequency mixing. We interpret qualitatively the numerical results obtained previously [11]. The method also enables us to make predictions concerning phase matching in other, possibly more optimized, geometrical conditions. These predictions are eventually compared to results from propagation calculations.

The quantum-path analysis method is presented in section 2, and the graphical phase matching in section 3. A summary is given in section 4 . 


\section{Quantum-path analysis of harmonic generation and frequency-mixing processes}

\subsection{Method}

As discussed above, the multi-quantum path dipole for the considered harmonic can be modelled as

$$
d(I)=\sum_{i} A_{i} \mathrm{e}^{\left(a_{i}-\mathrm{i} \alpha_{i}\right) I}
$$

where the index $i$ runs over all the paths considered. $A_{i}$ represents the weight of the different paths, $a_{i}$ is a growth parameter and $\alpha_{i}$ is called the reciprocal coefficient of the intensity (see below).

To separate the different quantum paths is not an easy task: up to now, it has been done only in the context of the SFA $[4,11]$, using a saddle-point approximation to obtain an expression like equation (1) analytically. This cannot be extended to more exact approaches, where the dipole moment is obtained numerically by solving the time-dependent Schrödinger equation. We therefore need a method to disentangle and characterize the quantum paths, directly from the numerical dipole data.

Our method takes advantage of the similarity between the intensity dependence of the phase of a dipole moment (equation (1)), and the time dependence of the phase of multicomponent exponentially decaying signals $s(t)$, as encountered for instance in nuclear magnetic resonance:

$$
s(t)=\sum_{i} A_{i} \mathrm{e}^{-\left(\lambda_{i}+\mathrm{i} \omega_{i}\right) t} .
$$

In equation (2), the index $i$ also runs over the different components, and the attenuation coefficient $\lambda_{i}$ and angular frequency $\omega_{i}$ have replaced the $a_{i}$ and $\alpha_{i}$ coefficients from equation (1). In the first case, the amplitudes of the individual components are increasing functions of intensity, while they are decreasing functions of time in the second case. However, we can easily invert the dipole data, considering them as a decreasing function of $-I$. We then analyse the phase of the high-harmonic generation/high-order frequency mixing (HHG/HFM) dipoles, by applying some of the powerful numerical tools designed to separate the different spectral components of a time-domain nuclear magnetic resonance signal [12].

Many algorithms have been developed with this aim, such as Fourier analysis, linear prediction methods and least-squares fitting. The older Fourier analysis method has the important advantage of being very simple to implement, but suffers from a well known drawback: the Fourier transform of a decaying exponential component is a complex LorentzDebye curve, with tails decaying slowly as $1 / \omega$ in magnitude, so that different spectral peaks often overlap, making the recovery of peak parameters a difficult task, and reducing the resolution [13]. We therefore use a modified Fourier method, known as derivative Fourier spectrometry, which is well known in the field of analytical chemistry [14-16]. In this method, the calculation of the spectrum is replaced by that of its $n$ th-order derivative. The magnitude of each resulting peak decreases away from the resonance frequency as $1 / \omega^{n+1}$, so that the peaks are much narrower, allowing previously unresolved peaks to become clearly observable. In practice, $n$ th-order derivation is easily achieved by multiplying the time-domain signal by $t^{n}$. This presents, however, the drawback of strongly enhancing the noise at long times. To cut off this noise, one uses an apodizing window, which can be of exponential form: $\exp \left(-\lambda_{\mathrm{w}} t\right)$, the windowing frequency $\lambda_{\mathrm{w}}$ being of the order of, but slightly less than the smallest characteristic frequency of the problem under consideration. One ends up with a 'derivating-apodizing' window, of the form

$$
W(t)=t^{n} \exp \left(-\lambda_{\mathrm{w}} t\right) .
$$


As shown in [17], in many cases, taking the derivation order $n=2$ often yields optimal results in terms of resolving power and signal-to-noise ratio. Other apodizing windows can also be used, such as Gaussian windows. However, these window functions usually result in lower resolutions than derivative-apodizing windows.

We now describe two possible methods that can be used to analyse any dipole moment of the form $|d(I)| \exp (\Phi(I))$, calculated numerically for a range of intensities between $I_{1}$ and $I_{2}$. In order to have the equivalent of decaying transients, we flip the intensity axis first, and simply replace the time variable by the flipped intensity variable. For this, we introduce an intensity parameter $I_{m}$, and make a change of variable: $I^{\prime}=I_{m}-I$.

In the first method, the dipole moment is multiplied by the apodizing window

$$
W\left(I^{\prime}\right)=I^{\prime 2} \exp \left(-a_{\mathrm{w}}^{0} I^{\prime}\right)
$$

and the resulting function is Fourier transformed, yielding a spectrum displaying one peak for each quantum path. The window parameter $a_{\mathrm{w}}^{0}$ that optimizes the resolution actually depends on the analysed function itself. The apodized dipole $d_{\mathrm{w}}$ reads

$$
d_{\mathrm{w}}\left(I^{\prime}\right)=\sum_{i} A_{i}^{\prime} I^{\prime 2} \exp \left(-\left(\left(a_{i}+a_{\mathrm{w}}^{0}\right)-\mathrm{i} \alpha_{i}\right) I^{\prime}\right)
$$

with $A_{i}^{\prime}=A_{i} \exp \left(\left(a_{i}-\mathrm{i} \alpha_{i}\right) I_{m}\right)$. In practice, the amplitude parameter $a_{i}$ is generally similar for all different quantum paths, but differs between the plateau and the cut-off regime. This allows us in either case to combine $a_{i}$ and $a_{\mathrm{w}}^{0}$ into a single window parameter $a_{\mathrm{w}}$. One ends up with

$$
d_{\mathrm{w}}\left(I^{\prime}\right)=\sum_{i} A_{i}^{\prime} \exp \left(\mathrm{i} \alpha_{i} I^{\prime}\right) I^{\prime 2} \exp \left(-a_{\mathrm{w}} I^{\prime}\right)
$$

$1 / a_{\mathrm{w}}$ gives the characteristic intensity span over which the Fourier transform is performed. According to the uncertainty relation, the resulting resolution on the reciprocal intensity determination is of the order of $a_{\mathrm{w}}$, and the reciprocal intensity obtained cannot be assigned to a particular intensity with a better precision than $1 / a_{\mathrm{w}}$. The choice of $a_{\mathrm{w}}$ therefore results from a trade-off between resolution in intensity and in reciprocal intensity, and has to be made first. Then, for a given extremum intensity $I_{m}$, one has to estimate the global growth parameter $a_{i}$ by a fit on the logarithm of the data. The window parameter $a_{\mathrm{w}}^{0}$ is finally obtained as $a_{\mathrm{w}}^{0}=a_{\mathrm{w}}-a_{i}$. This scheme gives satisfactory results for intensities within the plateau, but is more difficult to apply to the case of intensities close to the cut-off-plateau transition, or to the case of sumor difference-frequency mixing processes, for which the amplitudes of the different quantum paths turn out to vary strongly, as will be shown below.

The second method consists of analysing only the phase of the dipole, without taking into account its magnitude $\dagger$. The window parameter $a_{\mathrm{w}}$ can be chosen once for all. The Fourier transform is performed on the function,

$$
d_{\mathrm{w}}^{\phi}\left(I^{\prime}\right)=\mathrm{e}^{\mathrm{i} \Phi\left(I^{\prime}\right)} I^{\prime 2} \mathrm{e}^{-a_{\mathrm{w}} I^{\prime}}
$$

with $I^{\prime}=I_{m}-I$. In practice, this second method yields quite satisfactory results, and is much more flexible and easier to implement than the first one. In the following, we therefore present spectra obtained by the second method.

The intensity $I_{m}$ corresponds to the highest intensity for a given apodizing window. It can be varied from $I_{2}$ (highest intensity) down to typically $I_{1}+2 / \alpha_{\mathrm{w}}$. The windowed function is then Fourier transformed, which yields a spectrum displaying one or several well separated peaks.

$\dagger$ This type of approximation is actually common in Fourier transform studies. The only drawback is, sometimes, the appearance of weak satellite peaks at harmonic frequencies of the main peaks. It can be shown that an even cruder approximation, consisting of keeping only the sign of the real part of the dipole (and therefore reducing the phase information to +1 or -1 ) is often sufficient to recover the spectra [13]. 
These peaks correspond to quantum paths for the range of intensities for which the window function has large values, that is, for intensities around the abscissa $I_{\text {top }}$ of the peak of the apodizing window. One can therefore assign the reciprocal intensity coefficient $\alpha_{i}$ determined from a spectrum to its central intensity $I_{\text {top }}$, provided one bears in mind the uncertainty principle. If we translate the apodizing window in intensity, we therefore obtain the $\alpha_{i}$ coefficients as functions of $I_{\text {top }}$. Such a derivative Fourier spectrometry approach, used with a variable and translating window, has many analogies with a time-frequency representation. More precisely, it can be viewed as a Gabor-type analysis, $W\left(I^{\prime}\right)=I^{2} \exp \left(-\alpha_{\mathrm{w}} I^{\prime}\right)$ being the translated analysing function.

\subsection{Quantum-path analysis of $H H G$}

To check the validity of this method, we first examine the generation of the 45th harmonic of a $825 \mathrm{~nm}$ wavelength laser. The generating medium is a gas of neon atoms and the laser intensity varies between 0.3 and $10 \times 10^{14} \mathrm{~W} \mathrm{~cm}^{-2}$. This problem has been thoroughly analysed before, using the SFA $[6,18]$. We show in figure 1 the phase variation of the 45th harmonic component of the dipole moment. The phase decreases stepwise linearly as a function of the intensity of the driving laser field, with a slope of $-3.2 U_{\mathrm{p}} / \omega$ (where $U_{\mathrm{p}}$ is the ponderomotive potential, proportional to the laser intensity $I$ and to the square of the laser wavelength) in the cut-off region, and a slope of approximately $-6 U_{\mathrm{p}} / \omega$ in the plateau region. A saddle-point analysis of the dipole moment [6] shows that the latter slope is largely determined by the $\tau_{2}$ quantum path (we choose here to name the quantum paths by the label of the return time). Quantum interferences with the $\tau_{1}$ path are responsible for the fast variations superimposed onto the linear decrease; in contrast, the linear dependence in the cut-off regime is very smooth, as only one path contributes. At these low cut-off intensities, no classical trajectory exists that allows the electron to return to the ionic core with the required energy. Previous studies have shown that one has to allow for complex return times to determine the quantum-mechanical path [6]. At the cut-off-plateau transition, two relevant classical trajectories appear, giving rise to a sudden change in the atomic response behaviour, as demonstrated by the change of slope of the dipole phase.

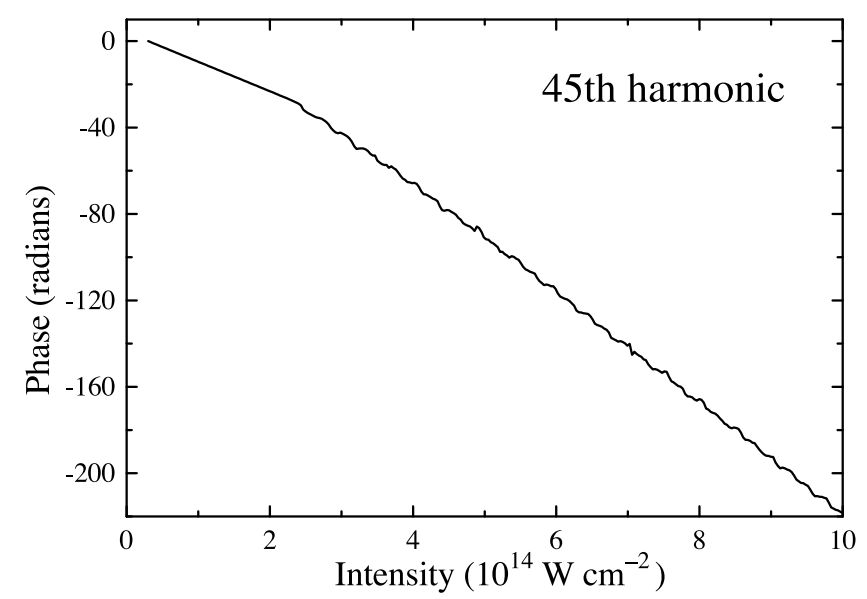

Figure 1. Intrinsic phase of the 45th harmonic of a $800 \mathrm{~nm}$ laser in neon, as a function of laser intensity, computed from the semiclassical model [4]. 

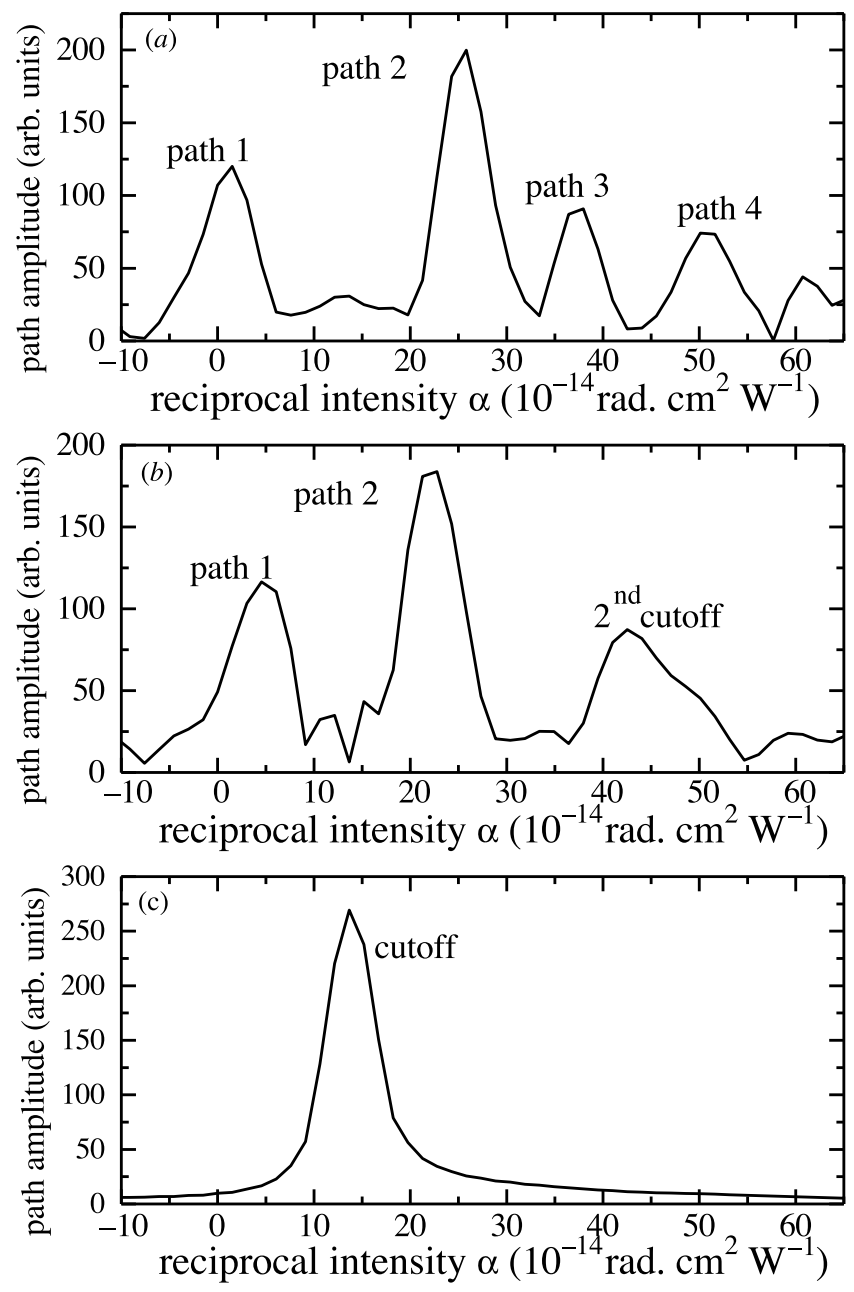

Figure 2. Fourier transforms of the 45 th harmonic phase $\Phi_{45}(I)$, calculated with apodizing functions centred at: (a) $I=6 \times 10^{14} \mathrm{~W} \mathrm{~cm}^{-2}$, (b) $I=3 \times 10^{14} \mathrm{~W} \mathrm{~cm}^{-2}$ and (c) $I=2 \times 10^{14} \mathrm{~W} \mathrm{~cm}^{-2}$. The abscissa corresponds to the reciprocal intensity coefficient $\alpha$.

In figure 2 we present several reciprocal-intensity spectra of the 45 th harmonic phase displayed previously. Figure 2(a) shows the spectrum obtained for $I_{m}=I_{2}=6.7 \times$ $10^{14} \mathrm{~W} \mathrm{~cm}^{-2}$. The spectrum is dominated by two peaks, readily seen to correspond exactly to the two slopes $\alpha_{1}, \alpha_{2}$ of the dominant quantum paths, with return times $\tau_{1}$ and $\tau_{2}$. The central values of $\alpha_{1}$ and $\alpha_{2}$ corresponding to those peaks are $1.3 \times 10^{-14}$ and $25.5 \times 10^{-14} \mathrm{~cm}^{2} \mathrm{~W}^{-1}$, respectively, in good agreement with the values resulting from a saddle-point analysis [4], namely, $1.4 \times 10^{-14}$ and $25.7 \times 10^{-14} \mathrm{~cm}^{2} \mathrm{~W}^{-1}$. Smaller peaks with larger $\alpha$ values can be interpreted as resulting from electron trajectories in the continuum with longer return times. Due to quantum diffusion, these trajectories have much smaller amplitudes [6], as is also shown here. These additional quantum paths arise in the socalled 'deep' plateau regime, when the intensity $I$ is clearly above the transition intensity between the plateau and the cut-off regions. Indeed, for the parameters used, the window 
function $W$ has a maximum for $I_{\text {top }}=6 \times 10^{14} \mathrm{~W} \mathrm{~cm}^{-2}$, which is well into the plateau regime.

It is interesting to displace the window to lower intensities (by decreasing $I_{m}$ ), so that its maximum comes closer to the end of the plateau, or even reaches the cut-off region. Such spectra are shown in figures $2(b)$ and $(c)$, respectively. In figure $2(b)$ two dominant quantum paths are still present, but the third and fourth paths have merged into a single peak. In figure 2(c), obtained for a window centred in the cut-off, the first two peaks have merged into a single one, in agreement with the results of the saddle-point analysis [6], and all higher ones have disappeared. The quantum paths therefore turn out to arise by pairs, at specific intensities labelled as cut-off intensity, second cut-off intensity (corresponding to the intensity chosen in figure $2(b))$, etc.

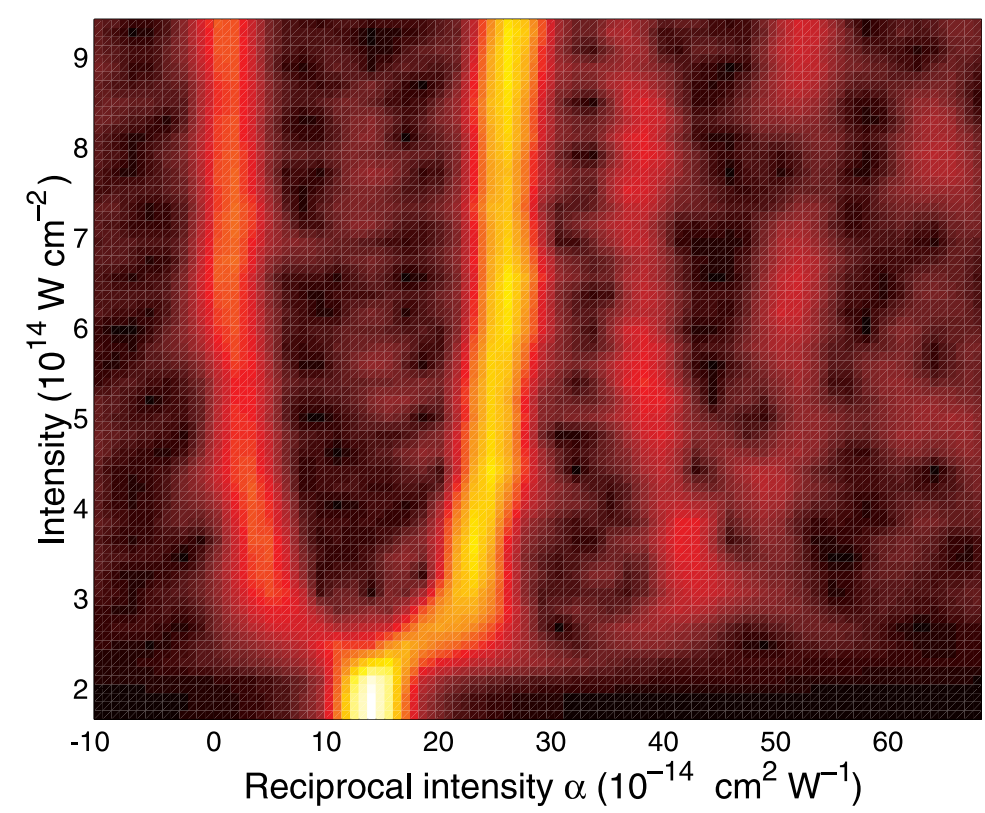

Figure 3. Pseudo-colour plot of the phase behaviour of the 45th harmonic in the (intensity)/(reciprocal intensity) representation. The abscissa corresponds to the reciprocal intensity coefficient $\alpha$. The greyscale gives the magnitude of successive Fourier transforms of the harmonic intrinsic phase as a function of intensity.

If we now use a large number of window positions to finely span the intensity scale, and plot in a white-greyscale the magnitude of the spectrum, as a function of reciprocal intensity $\alpha$ on the abscissa, and of the central intensity of the window on the ordinate, we obtain a full three-dimensional representation of the Fourier transform of the dipole phase, shown in figure 3. It gives a particularly illustrative view of the transition between the cut-off and the plateau, with a characteristic 'tuning-fork' shape. The ratio between the two main quantum paths can be estimated qualitatively, with a stronger second path in this case. The appearance of additional quantum paths, with longer return times, gives rise to a second tuning fork, centred 
on a reciprocal intensity parameter of $45 \times 10^{-14} \mathrm{~cm}^{2} \mathrm{~W}^{-1}$, and appearing at the second cut-off intensity of $3 \times 10^{14} \mathrm{~W} \mathrm{~cm}^{-2}$. Contributions from even higher quantum paths can also be noticed, but are very faint. All these features are in complete agreement with the saddle-point analysis.

We can therefore proceed to the case of an HFM processes, for which a saddle-point analysis of the dipole moment is much more difficult to implement. We first give the background for these frequency-mixing studies.

\subsection{An introduction to high-order frequency-mixing processes}

High-order frequency mixing in a two-colour laser field has recently attracted a lot of attention [11,19-21]. It offers the possibility to control, to some extent, the generation process $[19,21]$. When the fundamental laser field is mixed with its second harmonic, it can even lead to harmonic generation as well as an increased conversion efficiency for odd-harmonic generation. If the second field is tunable $[11,20]$, it can lead to the generation of tunable shortpulse XUV radiation, which is of great interest for applications. Recently [10], Gaarde et al studied high-order sum- and difference-frequency mixing processes in several gases (xenon, argon and neon). The experiment was performed by mixing the light from a fixed-frequency intense Ti:sapphire laser and the weaker tunable radiation from an optical parametric generator. Tunable radiation up to an energy of $70 \mathrm{eV}$ was obtained through mixing processes involving absorption or emission of one or two photons from the weak tunable field. This experimental work was followed by a theoretical study [11], using a strong-field approximation for describing the interaction between the atom and the strong laser field, and a perturbative approach for describing the effect of the second (weak) field on the system atom + strong field, up to second order. The high-order frequency-mixing dipole moments obtained were used as an input into a propagation calculation based on the slowly varying envelope approximation, with macroscopic parameters (such as gas-jet length and density, laser focusing conditions, etc) as close as possible to those of the experiment. Figure 4 shows a calculated spectrum in neon, using intensities of $3.1 \times 10^{14} \mathrm{~W} \mathrm{~cm}^{-2}$ for the strong field with a wavelength of $790 \mathrm{~nm}$, and $10^{11} \mathrm{~W} \mathrm{~cm}^{-2}$ for the weak field with a wavelength of $500 \mathrm{~nm}$. The sum-frequency processes, involving absorption of one photon from the weak field, are represented by + symbols, whereas the difference-frequency processes, involving emission of one photon from the weak field, are shown by squares.

In the present work we shall concentrate on two of these frequency-mixing processes, indicated by the arrows in figure 4 , namely ' $22+1$ ' sum-frequency mixing, involving net absorption of 22 photons from the intense field and one from the weak field, and ' $22-1$ ' difference-frequency mixing involving absorption of 22 photons from the intense field and emission of one to the weak field.

In figure 5, we show the variation with intensity of the dipole phases for these processes. The $22+1$ process is shown by a broken curve, and the $22-1$ process by a full curve. The fact that the phase of the sum-frequency mixing process decreases more rapidly with the laser intensity in the plateau region than that of the difference-frequency mixing process indicates that quantum paths with longer return times should play a more important role for the former than for the latter process. Moreover, interference effects between quantum paths seem more important in the sum-frequency process, whose phase curves display stronger oscillations and departures from a straight line. The plateau region starts at slightly higher intensity for the ' $22+1$ ' process than for ' $22-1$ ', because the photon energy is higher. 


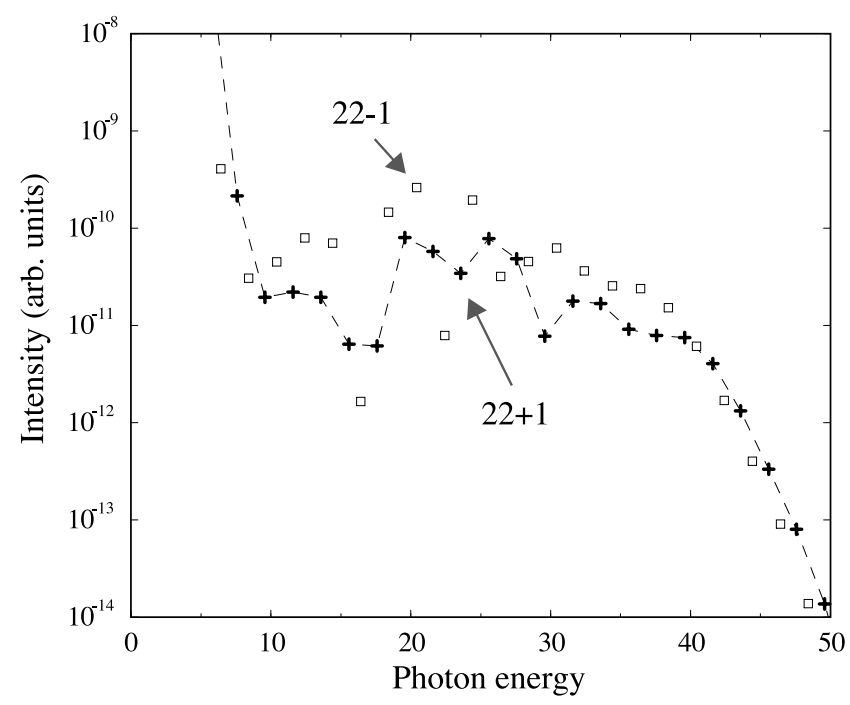

Figure 4. Wave-mixing peak intensities after propagation through a gas jet of neon, for one-photon sum-frequency (+) and difference-frequency (squares) processes, as a function of photon energies in units of $\hbar \omega_{1}$. The intensities and wavelengths of the fields are $I_{1}=3.1 \times 10^{14} \mathrm{~W} \mathrm{~cm}^{-2}$, $\lambda_{1}=790 \mathrm{~nm}$, and $I_{2}=10^{11} \mathrm{~W} \mathrm{~cm}^{-2}, \lambda_{2}=500 \mathrm{~nm}$.

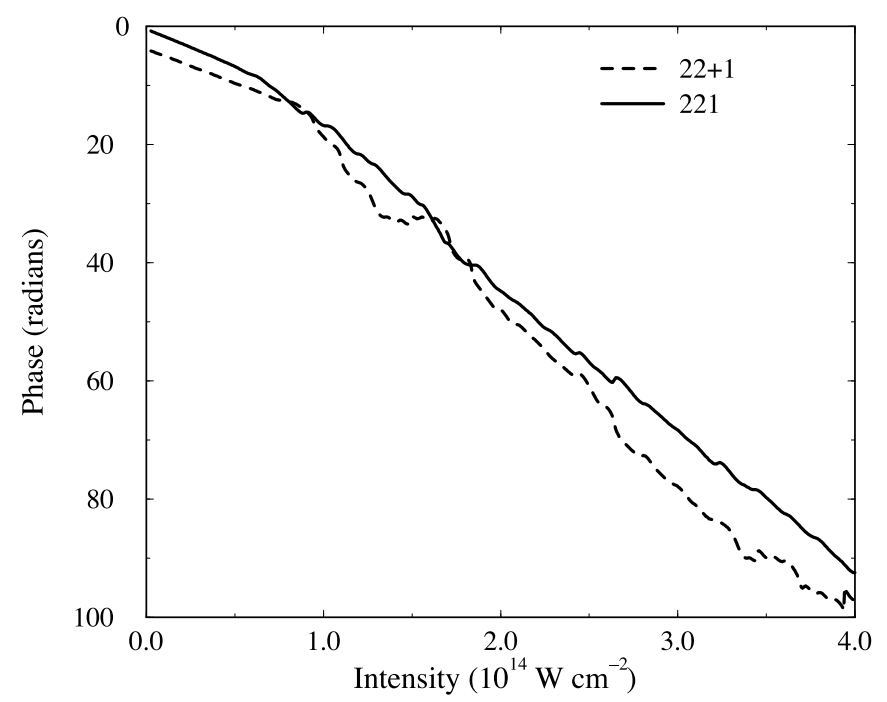

Figure 5. The dipole phase for the processes $22+1$ (broken curve) and $22-1$ (full curve) as a function of the intensity of the strong field.

\subsection{Quantum-path analysis of high-order frequency mixing processes}

Figures 6 and 7 present the result of the numerical quantum-path analysis method for the difference-frequency process ' $22-1$ ' and for the sum-frequency process ' $22+1$ ', respectively. Both exhibit significant differences from the results that can be obtained for a one-colour harmonic, as shown, for example, for the 45th harmonic in figure 3. The 'tuning-fork' 


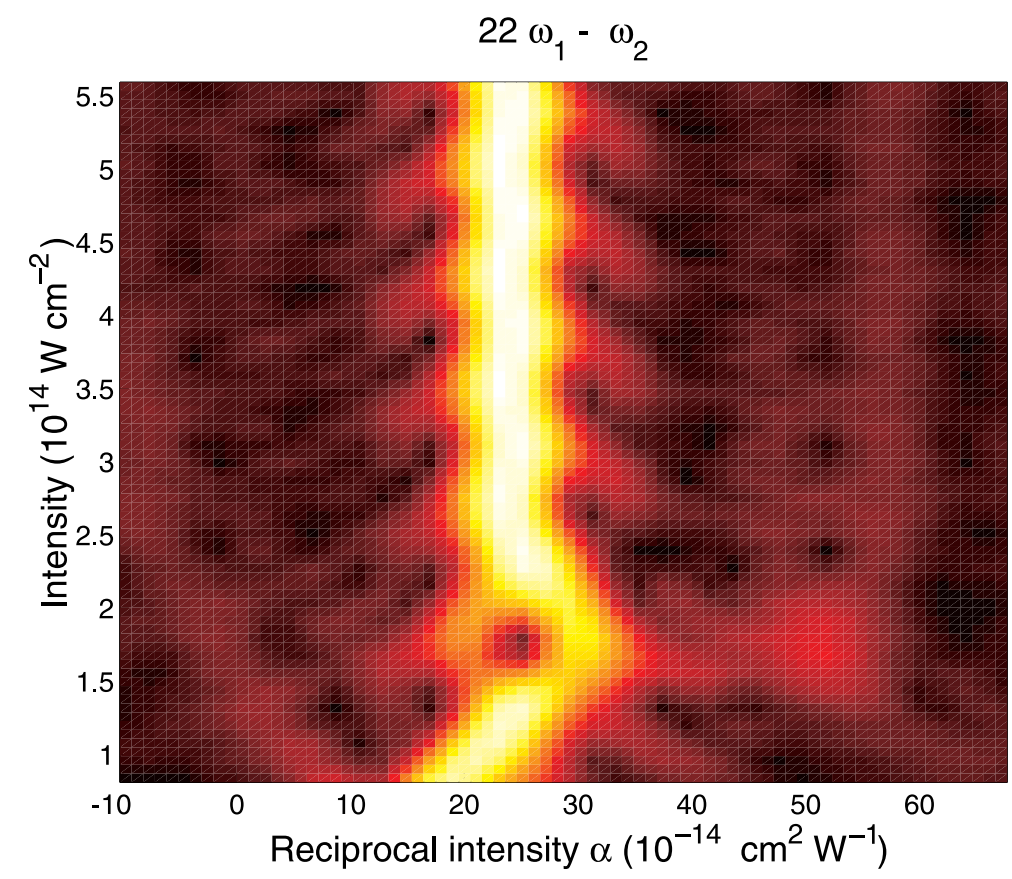

Figure 6. Pseudo-colour plot of the phase behaviour of the difference frequency process $22 \omega_{1}-\omega_{2}$ in the (intensity)/(reciprocal intensity) representation, obtained by a sliding-window Fourier transform analysis.

shape can barely be seen, simply because the plateau-cut-off transition happens just above $\simeq 8 \times 10^{13} \mathrm{~W} \mathrm{~cm}^{-2}$, which is the lowest intensity that the method can analyse.

In figure 6 , we observe essentially the contribution from the $\tau_{2}$ quantum path: the $\tau_{1}$ quantum path is vanishing at all intensities. This is in sharp contrast to the case of a singlecolour harmonic, for which the $\tau_{1}$ quantum path keeps a constant amplitude, approximately half as high as that of the $\tau_{2}$ quantum path (see figure 3 ). Finally, one can notice the appearance of a third quantum path, around $1.5 \times 10^{14} \mathrm{~W} \mathrm{~cm}^{-2}$, that does not survive to higher intensities.

In figure 7 , at $\simeq 1.1 \times 10^{14} \mathrm{~W} \mathrm{~cm}^{-2}$, the dominant path corresponds to a much higher reciprocal intensity than $\tau_{1}$ and $\tau_{2}(\alpha \approx 40)$. It splits into two branches (which we label as $\tau_{3}$ and $\left.\tau_{4}\right)$ at slightly higher intensity. The $\tau_{1}$ is dominant between $1.5 \times 10^{14}$ and $2 . \times 10^{14} \mathrm{~W} \mathrm{~cm}^{-2}$, and the $\tau_{2}$ path, from $2.2 \times 10^{14} \mathrm{~W} \mathrm{~cm}^{-2}$, up to higher intensities, except around $4 \times 10^{14} \mathrm{~W} \mathrm{~cm}^{-2}$.

As expected from the phase variations (figure 5), we see that quantum paths with longer return times, namely the $\tau_{3}, \tau_{4}$ paths, contribute significantly to the $22+1$, and not to the $22-1$ process. Note that there are, however, regions of intensity where the slow phase behaviour $\left(\tau_{1}\right)$ is dominant for the $22+1$ process. This should induce different phase-matching conditions depending on the intensity.

These intensity-dependent features were found in [11] to correspond to quantum interferences characteristic of sum- or difference-frequency processes. Indeed, a sumfrequency process involves absorption of, for instance, $22 \omega_{1}$ photons and one $\omega_{2}$ photon. Along a given electron trajectory, absorption of the latter $\omega_{2}$ photon can arise only in the vicinity of the nucleus, which is possible either at the time of tunnelling or of recombination. 


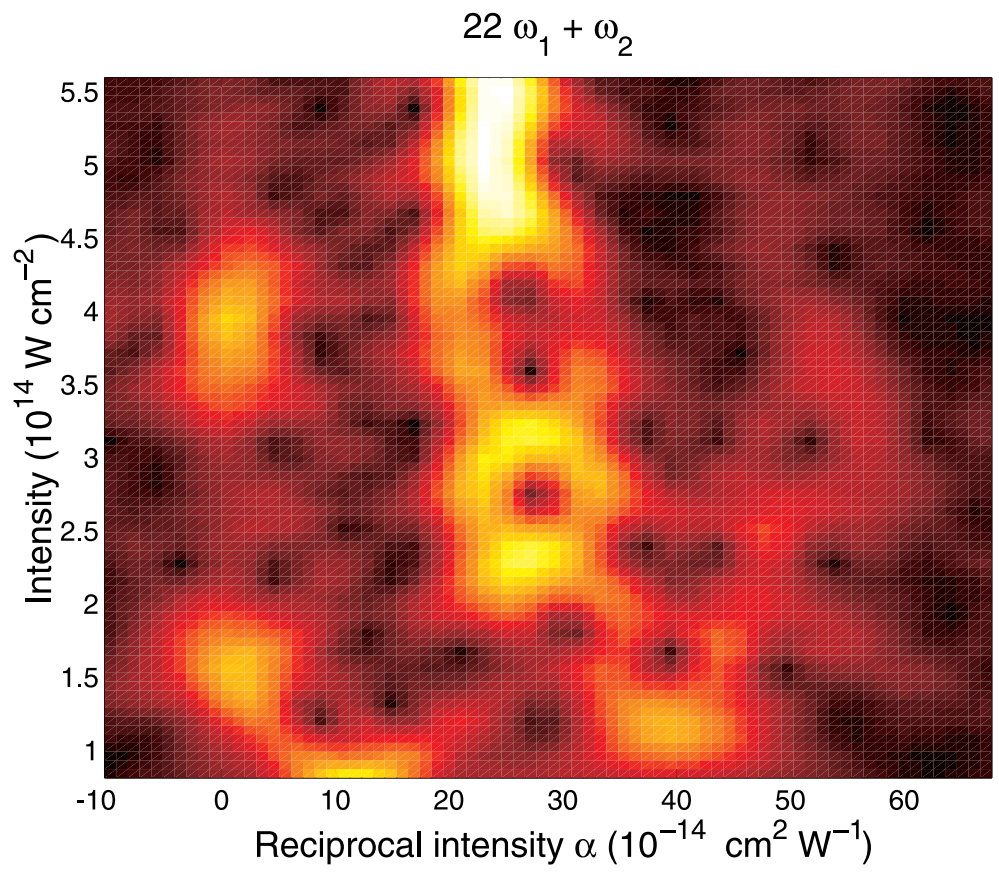

Figure 7. Pseudo-colour plot of the phase behaviour of the sum-frequency process $22 \omega_{1}+\omega_{2}$ in the (intensity)/(reciprocal intensity) representation.

As a result, each quantum path actually splits into two paths, that interfere constructively or destructively depending, in particular, on the intensity, yielding the variations in quantum-path amplitudes displayed in figure 7. A study of neighbouring wave-mixing peaks shows that such phase behaviours are generic, so that most of the conclusions on phase matching can be generalized to other sum- or difference-frequency processes. However, for some specific orders, quantum interference effects happen to induce very effective cancellations between the different quantum paths contributing to the dipole moment, which results in a strong decrease of the overall efficiency, as shown in figure 4 by the poor yield of the ' $24-1$ ' process.

Finally, it is interesting to compare these phase behaviours with the dipole strengths as a function of intensity, displayed in figure 8 . Both sum and difference processes increase in parallel up to $2.5 \times 10^{14} \mathrm{~W} \mathrm{~cm}^{-2}$, from which the difference-frequency process exhibits a steeper variation with intensity. This implies that the atomic response is enhanced by almost an order of magnitude whenever the $\tau_{2}$ path is dominant.

\section{Phase-matching analysis of high-order harmonic generation and frequency-mixing processes}

\subsection{Description of the graphical method}

We first briefly describe the graphical method used to study the phase-matching conditions in the nonlinear medium. It is described in detail in [9]. To achieve phase matching, it 


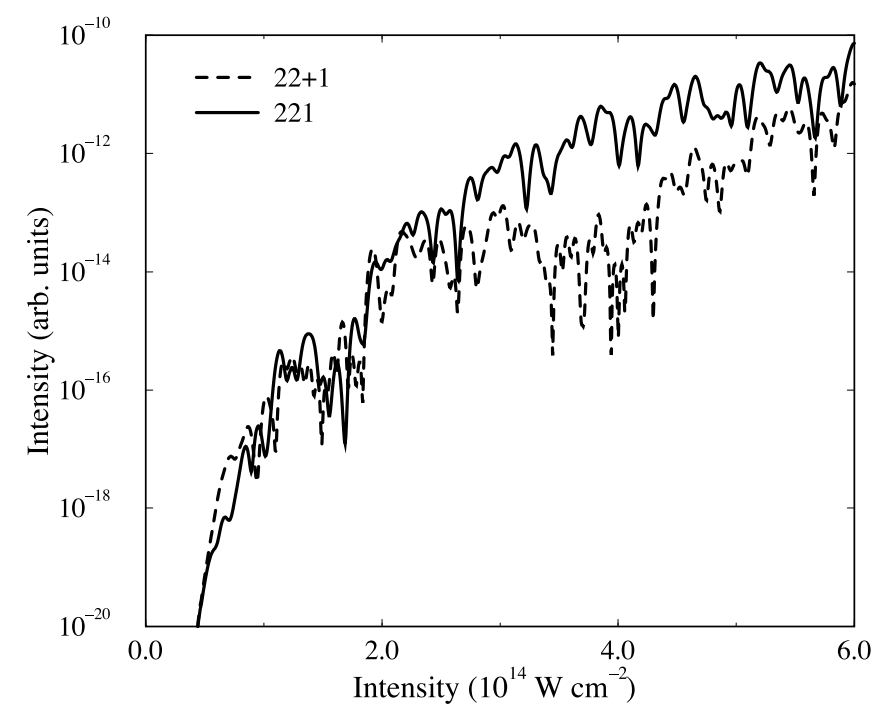

Figure 8. The dipole strengths for the processes $22+1$ (broken curve) and $22-1$ (full curve) as functions of the intensity of the strong field.

is necessary to conserve momentum, which can be expressed by the following vectorial relation:

$$
\vec{k}_{q}=q \vec{k}_{1}+\vec{K}
$$

$\vec{k}_{q}$ represents the wavevector of the generated field, $\vec{K}(r, z)=\vec{\nabla} \Phi_{\mathrm{at}}(r, z)$ is the effective wavevector due to the spatial variations of the dipole phase $\Phi_{\text {at }}$, and $\vec{k}_{1}(r, z)=$ $\left(n_{1} \omega / c\right) \vec{e}_{z}+\vec{\nabla} \Phi_{\text {foc }}$ is the wavevector of the fundamental field, including the effect of focusing. Here $\vec{e}_{z}$ is the unit vector in the propagation direction, $\Phi_{\text {foc }}$ is the focusinginduced phase of a Gaussian beam (reducing to the Gouy phase on axis), and $n_{1}$ is the local index of refraction at the laser frequency, including the effects of atoms and electrons.

Since $\vec{k}_{1}$ and $\vec{K}$ vary throughout the focal volume, equation (8) can never be fulfilled exactly throughout the focal region. Phase matching can be assessed more quantitatively by examining the wavevector mismatch $\vec{\delta} k=\vec{k}_{q}-\left(q \vec{k}_{1}+\vec{K}\right)$. Good phase matching corresponds to a locally small value of $|\delta k|$ with respect to the inverse of a typical length of a gas jet, while poor phase matching corresponds to a larger $|\delta k|$.

The graphical method described in [9] consists of representing in greyscale the distribution of $|\delta k|$ in the focal volume. As an example, we show in figure 9 a phase-matching map for the 45 th harmonic, where the dipole phase is taken to be that of the $\tau_{2}$ quantum path. For the sake of simplicity, the effect of free electrons is neglected in this example, but could be easily included provided one defines the ionization probability distribution. The plot uses cylindrical coordinates $(r, z)$. White and light-grey shading indicate areas where phase matching is good; dark grey indicates poor phase matching. The full curves are contours of equal intensity. This map contains three regions of good phase matching, two large off-axis lightgrey areas corresponding to non-collinear phase matching, connected by a central thin strip that corresponds to collinear phase matching on axis. For the latter position, the wavevector induced by the atomic phase $\vec{K}$ compensates exactly the wavevector mismatch $\vec{k}_{q}-q \vec{k}_{1}$ due to focusing and possibly to free electrons. On the right-hand side, $\vec{K}$ overcompensates the phase 


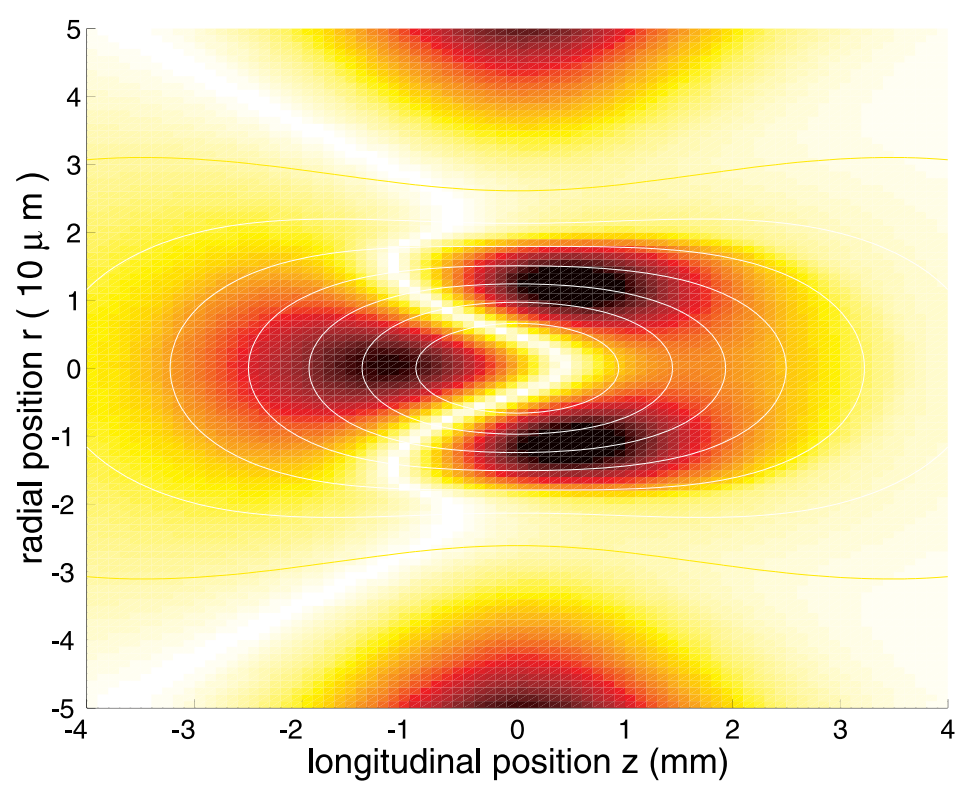

Figure 9. Pseudo-colour phase-matching map of the 45th harmonic, at an intensity of $I=$ $6 \times 10^{14} \mathrm{~W} \mathrm{~cm}^{-2}$. Only the dominant second quantum path is considered. The laser propagates from left to right, on a central axis centred in the figure. Contour lines delineate the laser intensity levels. Shading indicates the residual phase mismatches: from locally perfect phase matching (white) to strong phase mismatches (black, $50 \mathrm{~cm}^{-1}$ ).

mismatch; whereas on the left-hand side, it is either insufficient to compensate it, or it makes it even worse, thus strongly deteriorating the phase matching.

For a given focusing geometry, a phase-matching map depends both on the peak intensity and on the quantum path considered, through the parameter $\alpha I_{0}$ (atomic phase at the peak intensity).

\subsection{Phase-matching analysis of high-order frequency mixing processes}

We can now apply this graphical method to the case of the high-order frequency mixing processes discussed previously. Figures 10 and 11 show the results obtained for the ' $22-1$ ' and ' $22+1$ ' processes, respectively. The conditions are chosen to be the same as in [11]: for the intense laser field, we have $I_{1}=3.1 \times 10^{14} \mathrm{~W} \mathrm{~cm}^{-2}$, and $b_{1}$ (confocal parameter) $=5 \mathrm{~mm}$. For the weak dressing field, $b_{2}=50 \mathrm{~mm}$, and it is assumed to be focused at the same position as the strong field. Since we compare the two processes within the perturbative regime with respect to the weak dressing field, with the same order of nonlinearity, the intensity of this second field does not come into play and does not need to be specified. The plots are obtained using the complete dipole phase variation, thus including all quantum paths. When two (or more) quantum paths are comparable, they interfere and lead to pronounced ripples in the phase-matching maps. The full curves are iso-intensity contours delimiting intensity regions of dominant quantum paths. Note, in particular, the region where the $\tau_{3}$ quantum path is dominant in figure 10 , and the $\tau_{1}$ quantum path is dominant in figure 11 . 


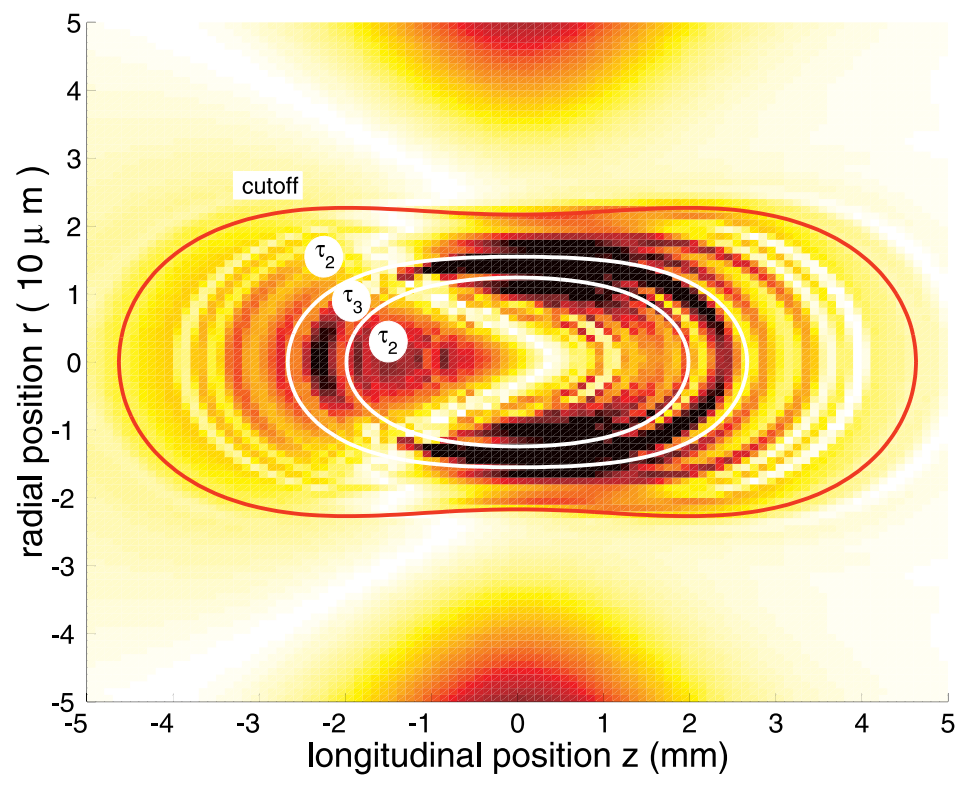

Figure 10. Pseudo-colour phase-matching map of the difference-frequency process $22 \omega_{1}-\omega_{2}$, for an intensity $I=3.1 \times 10^{14} \mathrm{~W} \mathrm{~cm}^{-2}$. Full curves and labels indicate the area for which the atomic response is in the cut-off regime (i.e. has an exponentially decaying amplitude when the laser intensity decreases away from the focal point), and those for which the $\tau_{2}$ and $\tau_{3}$ quantum paths prevail.

Both phase-matching maps illustrate a relatively good phase-matching region when the gas medium is centred at the laser focus (around $z=0$ ), or slightly to the right. It is especially good for the difference-frequency process, and corresponds to the optimization of phase matching for the $\tau_{2}$ quantum path. The two maps differ significantly in the intensity region close to $z= \pm 2 \mathrm{~mm}$. Much better phase matching is predicted for the sum- than for the differencefrequency process. This is due to the large amplitude of the first quantum path for the sumfrequency process, while the difference-frequency process remains dominated by a longer quantum path $\left(\tau_{3}\right)$. For the latter process, the effective wavevector due to the atomic phase adds to that induced by focusing, leading to poor phase-matching conditions. It is almost negligible for the sum process, and the phase-matching conditions are much better.

To check these qualitative predictions, we have performed numerical propagation calculations, similar to those presented in [11]. We show in figures 12 and 13 the intensity of the XUV light generated by both processes, as a function of the intensity of the first (intense) field, for the two positions of the centre of the gas jet $(z=0,-2 \mathrm{~mm})$ relative to the laser focus (located at zero). Clearly, the difference-frequency process dominates in the $z=0$ case, as noticed by Gaarde et al [11], whereas the sum and difference processes yield almost identical intensities in the $z=-2 \mathrm{~mm}$ case. Considering that the single-atom response for the sum-frequency process is lower than that of the difference-frequency process in that range of intensities, we note a remarkable effect of propagation and phase matching: the difference-frequency process is impaired by a poorer phase matching, while the sum-frequency process is enhanced enough to compensate for the difference in the single-atom response. This 


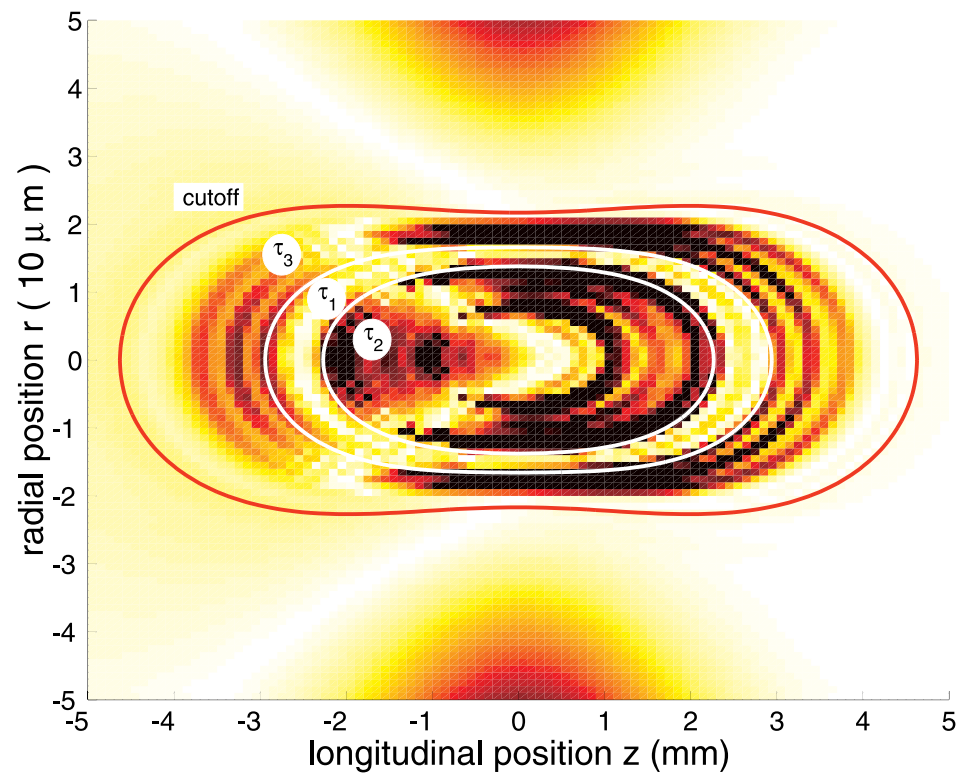

Figure 11. Pseudo-colour phase-matching map of the sum-frequency process $22 \omega_{1}+\omega_{2}$, for an intensity $I=3.1 \times 10^{14} \mathrm{~W} \mathrm{~cm}^{-2}$. Full curves and labels indicate the area for which the atomic response is in the cut-off regime, and those for which the $\tau_{3}, \tau_{1}$, and $\tau_{2}$ quantum paths prevail.

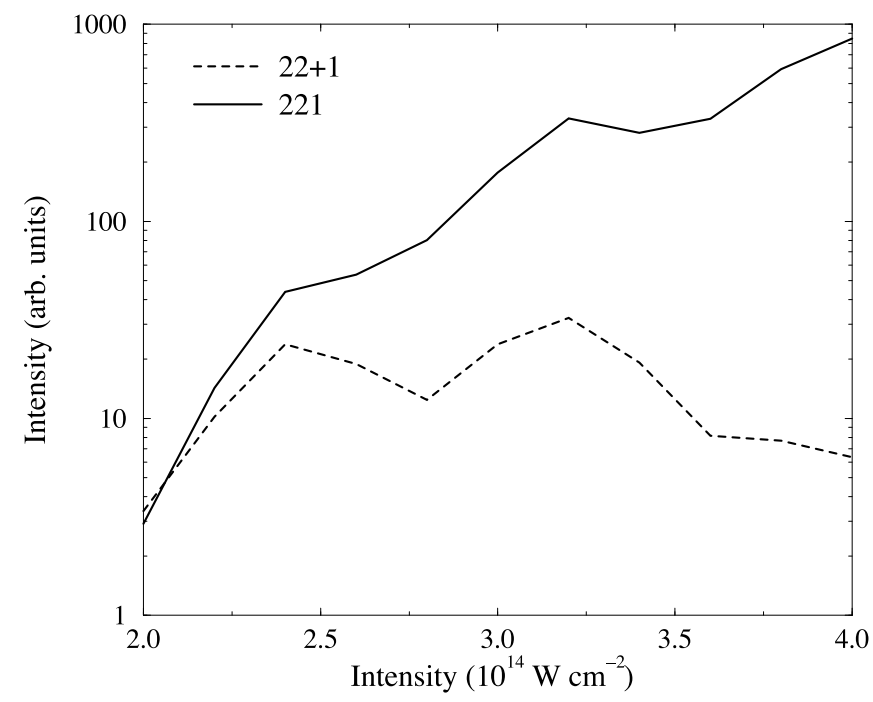

Figure 12. Yields of the sum (' $22+1$ ', broken curve) and difference (' $22-1$ ', full curve) frequency processes, as a function of laser intensity, for a gas jet located exactly at focus.

enhancement illustrates the potential of the phase-matching map method to obtain guidance about which geometrical conditions one should use to optimize a particular process. 


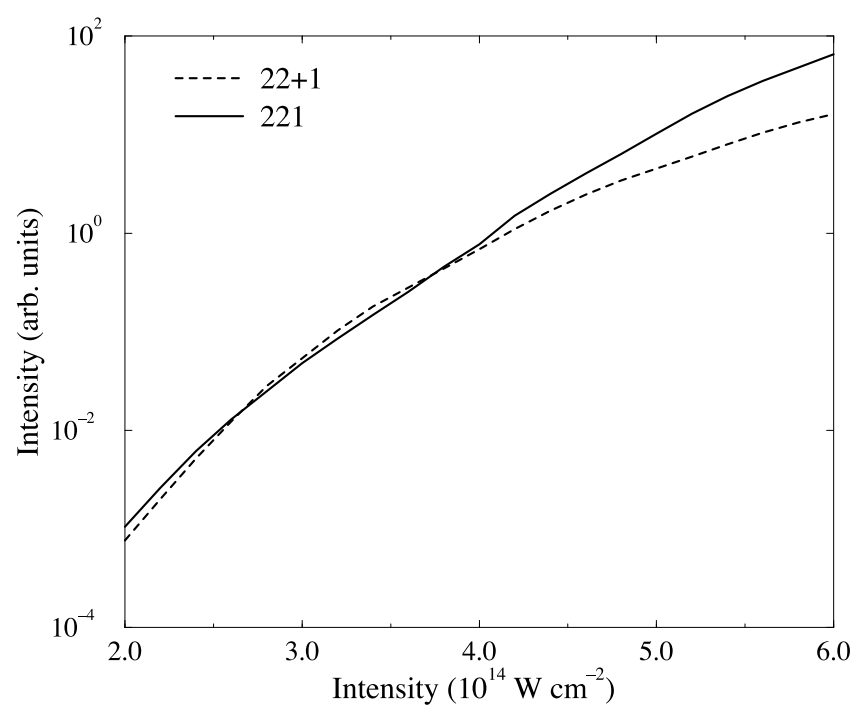

Figure 13. Yields of the sum (' $22+1$ ', broken curve) and difference (' $22-1$ ', full curve) frequency processes, as a function of laser intensity, for a gas jet located at the optimal position determined from figure 11 .

\section{Conclusion}

The previous example shows how the graphical phase-matching map method can be used to pinpoint the conditions for which phase matching for a given process will be optimized. These maps can be calculated by a standard personal computer in just a few seconds, whereas complete propagation calculations using standard Crank-Nicholson algorithms would take considerably longer. This may turn out to be crucial in practice, as the space of parameters to be chosen in an experiment is often far too large to allow systematic numerical investigations prior to experiment, whereas the present method lends itself much more to such a preliminary enquiry.

In addition, we have shown that the quantum-path analysis method can be a useful tool to investigate the single-atom response, as well as to interpret macroscopic phase-matching results. This method, in particular, was used recently to explain the different spatial and temporal coherence properties of high-harmonic radiation, for the two main quantum paths [22]. It constitutes, therefore, a major step in linking the microscopic and macroscopic features of high-order nonlinear processes in strong laser fields.

\section{Acknowledgment}

One of the authors $(\mathrm{PhB})$ would like to thank his father Dr Yves Balcou for decisive help in the field of numerical spectrometric methods.

\section{References}

[1] L'Huillier A et al 1995 J. Nonlinear Opt. Phys. Mater. 4 647-65

[2] Corkum P B 1993 Phys. Rev. Lett. 71 1994-7

[3] Schafer K J, Yang B, DiMauro L F and Kulander K C 1993 Phys. Rev. Lett. 70 1599-602 
[4] Lewenstein M, Balcou Ph, Ivanov M Yu, L'Huillier A and Corkum P 1994 Phys. Rev. A 49 2117-32

[5] Becker W, Lohr A, Kleber M and Lewenstein M 1997 Phys. Rev. A 56 645-56

[6] Lewenstein M, Salières P and L'Huillier A 1995 Phys. Rev. A 52 4747-54

[7] Antoine Ph, L'Huillier A and Lewenstein M 1996 Phys. Rev. Lett. 77 1234-7

[8] Antoine Ph, Milosević D B, L'Huillier A, Gaarde M B, Salières P and Lewenstein M 1997 Phys. Rev. A 56 4960-9

[9] Balcou Ph, Salières P, L'Huillier A and Lewenstein M 1997 Phys. Rev. A 55 3204-10

[10] Gaarde M B, Antoine Ph, Persson A, Carré B, L'Huillier A and Wahlström C-G 1996 J. Phys. B: At. Mol. Opt. Phys. 29 L163-8

[11] Gaarde M B, L'Huillier A and Lewenstein M 1996 Phys. Rev. A 54 4236-48

[12] Hoch J C and Stern A S 1996 NMR Data Analysis (New York: Wiley)

[13] Marshall A G and Verdun F-R 1990 Fourier Transforms in NMR, Optical and Mass Spectrometry: a User's Handbook (Amsterdam: Elsevier)

[14] Talsky G 1994 Derivative Spectrophotometry (Weinheim: VCH)

[15] Balcou Y 1994 Rapid Commun. Mass Spectrom. 8 942-4

[16] Balcou Y and Balcou Ph 1995 Proc. 13th Biennal. Coll. on Hertzian Optics and Dielectrics (Saragoza) ed J M Fornies-Marquina pp 160-3

[17] Kim H S and Marshall A G 1995 J. Mass Spectrom. 30 1237-44

[18] Salières P, L'Huillier A and Lewenstein M 1995 Phys. Rev. Lett. 74 3776-9

[19] Zuo T, Bandrauk A D, Ivanov M and Corkum P B 1995 Phys. Rev. A 51 3991-8

[20] Eichmann H, Meyer S, Riepl K, Momma C and Wellegehausen B 1994 Phys. Rev. A 50 R2834-6

[21] Preston S G and Watson J B 1998 J. Phys. B: At. Mol. Opt. Phys. 31 2247-57

[22] Gaarde M B, Salin F, Constant E, Balcou Ph, Schafer K J, Kulander K C and L'Huillier A 1999 Phys. Rev. A 59 $1367-73$ 